\title{
Adaptive Finite Element Method in Nanophotonic Simulations
}

\author{
L. Beilina ${ }^{1, b)}$, L. Mpinganzima ${ }^{2,3, a)}$ and P. Tassin ${ }^{3}$ \\ ${ }^{1}$ Department of Mathematical Sciences, Chalmers University of Technology and Gothenburg University, \\ 41296 Gothenburg, Sweden \\ ${ }^{2}$ Department of Applied Mathematics, University of Rwanda, P.O.Box 3900 Kigali, Rwanda \\ ${ }^{3}$ Department of Physics, Chalmers University of Technology and Gothenburg University, 41296 \\ Gothenburg, Sweden \\ a)Corresponding author: lydie.mpinganzima@chalmers.se \\ b)larisa@chalmers.se
}

\begin{abstract}
The problem of constructing nanophotonic structures of arbitrary geometry with prescribed properties was studied using an adaptive optimization algorithm. Stability estimates for the forward and adjoint problems involved in this algorithm are presented. A numerical example illustrates the construction of nanostructure in two dimensions.
\end{abstract}

\section{Introduction}

In this paper, we investigate the stability of a nanoparametric optimization algorithm presented in [4] for constructing nanophotonic structures of arbitrary geometry with prescribed properties. Examples of such structures are photonic crystals (structured in the wavelength scale), metamaterials (subwavelength structured media with new optical properties that are not available from natural materials) and plasmonic devices $[6,8,10]$. Our goal is to solve the Coefficient Inverse Problem (CIP) for the electromagnetic wave equation with unknown material distribution and known scattering properties. As shown in [4], the problem can be reformulated as an optimization problem for the Tikhonov functional which is minimized on locally adaptively refined meshes using the Lagrangian approach. In the current work, we present the stability, or energy estimates for the solutions of forward and adjoint problems, respectively. These estimates will involve the stability of the whole optimization procedure.

To solve our CIP we use the conjugate gradient method. It is well-known that gradient-like methods for minimization of the Tikhonov functional suffer from having multiple local minima and this leads to local convergence of these methods. In order to guarantee the global convergence, a starting point should be chosen in a small neighbourhood of the exact solution. Our numerical tests show that construction of a good initial guess is a main challenge in the adaptive optimization algorithm with applications to nanophotonic simulations. 


\section{Statement of the forward and inverse problems}

Let $D \subset \mathbb{R}^{2}$ be a convex bounded domain with a smooth boundary $\partial D$ such that $\partial D=\partial_{1} D \cup \partial_{2} D \cup \partial_{3} D$ where $\partial_{1} D$ and $\partial_{2} D$ are, respectively, top and bottom sides of the domain $D$, and $\partial_{3} D$ is the union of left and right sides of this domain. Denote by $D_{T}:=D \times(0, T), \partial D_{T}:=\partial D \times(0, T), T>0$. We also define $S_{1}:=\partial_{1} D \times(0, T), S_{2}:=\partial_{2} D \times(0, T)$ and $S_{3}:=\partial_{3} D \times(0, T)$. We consider that in $S_{1}$ we have timedependent backscattering observations. Let now $x=\left(x_{1}, x_{2}\right)$ denote a point in $D$ and introduce the following spaces of real valued functions

$$
\begin{aligned}
H_{u}^{1}\left(D_{T}\right) & :=\left\{w \in H^{1}\left(D_{T}\right): w(\cdot, 0)=0\right\}, H_{\lambda}^{1}\left(D_{T}\right):=\left\{w \in H^{1}\left(D_{T}\right): w(\cdot, T)=0\right\}, \\
U^{1} & =H_{u}^{1}\left(D_{T}\right) \times H_{\lambda}^{1}\left(D_{T}\right) \times C(\bar{D}) .
\end{aligned}
$$

Thus, similarly to [4, 9], we consider the propagation of electromagnetic waves with a field polarization and model the wave propagation by the following scalar wave equation:

$$
\begin{cases}\varepsilon \frac{\partial^{2} u}{\partial t^{2}}-\Delta u=\delta\left(x_{2}-x_{0}\right) p(t) & \text { in } D_{T}, \\ u(x, 0)=f_{0}(x), \quad u_{t}(x, 0)=0 & \text { in } D, \\ \partial_{n} u=-\partial_{t} u & \text { on } S_{1}, \\ \partial_{n} u=-\partial_{t} u & \text { on } S_{2}, \\ \partial_{n} u=0 & \text { on } S_{3},\end{cases}
$$

where $u$ denote the electric field generated by the plane wave $p(t)$ which is incident at $x_{2}=x_{0}$ and propagates along the $x_{2}$ axis, $\varepsilon(x)$ is the spatially distributed dielectric permittivity function. Observe that we use in the above problem the first order absorbing boundary conditions.

For the computational solution of (2) we use the domain decomposition finite element/finite difference (FE/FD) method of [2]. This method has been applied to the solution of different CIPs for the acoustic wave equation in $[1,2]$. To apply the method of [2] we decompose $D$ into two regions $D_{F E M}$ and $D_{F D M}$ such that the whole domain $D=D_{F E M} \cup D_{F D M}$, and $D_{F E M} \cap D_{F D M}=\emptyset$. In $D_{F E M}$ we use the finite element method (FEM), and in $D_{F D M}$ we will use the Finite Difference Method (FDM).

The function $\varepsilon(x)$ in (2) belongs to the following set of admissible parameters

$$
M_{\varepsilon}=\left\{\varepsilon: \varepsilon(x) \in(0, M], M=\text { const. }>0 \forall x \in D_{F E M}, \varepsilon(x)=1 \forall x \in D_{F D M}\right\} .
$$

We consider the following Inverse Problem (IP): Let the coefficient $\varepsilon(x)$ in the problem $(2)$ satisfy conditions (3) and assume that $\varepsilon(x)$ is unknown in the domain $D \backslash D_{F D M}$. Determine the function $\varepsilon(x)$ in (2) for $x \in D \backslash D_{F D M}$, assuming that the following function $\widetilde{u}(x, t)$ is known

$$
u(x, t)=\widetilde{u}(x, t), \forall(x, t) \in S_{1} .
$$

\section{Optimization method}

In this section we present the reconstruction method to solve inverse problem IP. This method is based on the finding of the stationary point of the following Tikhonov functional

$$
F(u, \varepsilon)=\frac{1}{2} \int_{S_{1}}(u-\widetilde{u})^{2} z_{\delta}(t) d \sigma d t+\frac{1}{2} \gamma \int_{D}\left(\varepsilon-\varepsilon_{g}\right)^{2} d x,
$$


where $u$ satisfies the equations (2), $\varepsilon_{g}$ is the initial guess for $\varepsilon, \widetilde{u}$ is the observed field at $S_{1}, \gamma>0$ is the regularization parameter and $z_{\delta}$ can be chosen as in [3]. To find a minimum of (5) we use the Lagrangian approach as in $[1,3]$ and define the following Lagrangian

$$
L(v)=F(u, \varepsilon)+\int_{D_{T}} \lambda\left(\varepsilon \frac{\partial^{2} u}{\partial t^{2}}-\Delta u-\delta\left(x_{2}-x_{0}\right) p(t)\right) d x d t
$$

where $v=(u, \lambda, \varepsilon) \in U^{1}$, and search for a stationary point with respect to $v$ satisfying $\forall \bar{v}=(\bar{u}, \bar{\lambda}, \bar{\varepsilon}) \in U^{1}$

$$
L^{\prime}(v)(\bar{v})=0,
$$

where $L^{\prime}(v)(\bar{v})$ is the Jacobian of $L$ at $v$. The adjoint problem will be the following [1]:

$$
\begin{cases}\varepsilon \frac{\partial^{2} \lambda}{\partial t^{2}}-\Delta \lambda=-(u-\widetilde{u}) z_{\delta} & x \in S_{1} \\ \lambda(\cdot, T)=\frac{\partial \lambda}{\partial t}(\cdot, T)=0, & \\ \partial_{n} \lambda=\partial_{t} \lambda & \text { on } S_{1} \\ \partial_{n} \lambda=\partial_{t} \lambda & \text { on } S_{2} \\ \partial_{n} \lambda=0 & \text { on } S_{3}\end{cases}
$$

\section{Stability estimates}

Stability estimates for the forward problem (2) and adjoint problem (8) follow from the stability estimate of [2] and can be derived using the technique of [7]. The only difference reside in the integration in time; $(0, T)$ for the forward problem and $(T, t)$ for the adjoint problem, respectively. For the analysis we first introduce the $L_{2}$ inner product and the norm over $D_{T}$ and $D$, correspondingly, as

$$
((u, v))_{D_{T}}=\int_{D_{T}} u v d x d t,\|u\|_{L_{2}\left(D_{T}\right)}^{2}=((u, u))_{D_{T}},(u, v)_{D}=\int_{D} u v d x,\|u\|_{L_{2}(D)}^{2}=(u, u)_{D}
$$

We can prove the following stability estimates for the forward problem (2) and adjoint problem (8).

Theorem 1. Assume that condition (3) for the functions $\varepsilon(x)$ holds. For any $t \in(0, T)$ we define $D_{t}=$ $D \times(0, t)$. Assume that there exists a solution $u \in H^{2}\left(D_{T}\right)$ of the problem (2). Then $u$ is unique and there exists a positive constant $A=A\left(\|\varepsilon\|_{D}, t\right)$ such that the following energy estimate is true for every $t \in(0, T)$

$$
\left\|\sqrt{\varepsilon} \partial_{t} u(x, t)\right\|_{L_{2}(D)}^{2}+\|\nabla u(x, t)\|_{L_{2}(D)}^{2} \leq A\left[\left\|p(t) \delta\left(x_{2}-x_{0}\right)\right\|_{L_{2}\left(D_{t}\right)}^{2}+\left\|\nabla f_{0}\right\|_{L_{2}(D)}^{2}\right]
$$

Theorem 2. Assume that condition (3) for the functions $\varepsilon(x)$ holds. For any $t \in(T, 0)$ we define $D_{t_{a}}=$ $D \times(T, t)$. Assume that there exists a solution $\lambda \in H^{2}\left(D_{T}\right)$ of the problem (8) and a solution $u \in H^{2}\left(D_{T}\right)$ of the problem (2) which satisfy to the Theorem 1. Then $\lambda$ is unique and there exists a positive constant $B=B\left(\|\varepsilon\|_{D}, t\right)$ such that the following energy estimate is true for every $t \in(T, 0)$

$$
\left\|\sqrt{\varepsilon} \partial_{t} \lambda(x, t)\right\|_{L_{2}(D)}^{2}+\|\nabla \lambda(x, t)\|_{L_{2}(D)}^{2} \leq B\left\|(\tilde{u}-u) z_{\delta}\right\|_{L_{2}\left(D_{t a}\right)}^{2} .
$$




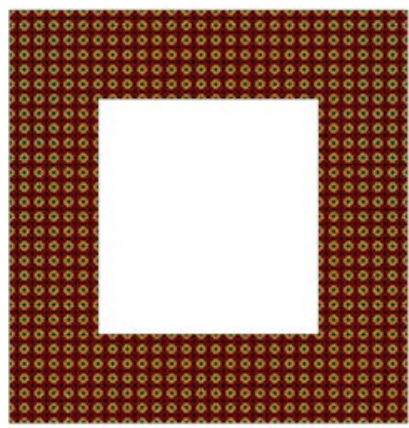

a) $\varepsilon_{g}=0.5$

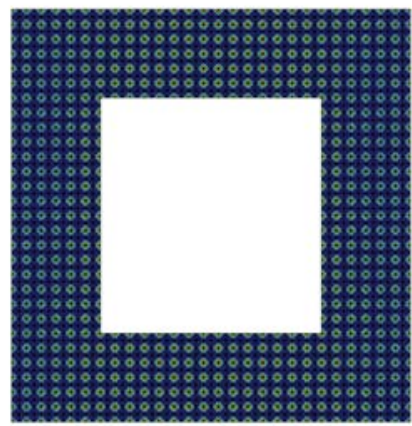

b) $\varepsilon_{g}=1.5$

FIGURE 1. Reconstructions in three times adaptively refined mesh for different $\varepsilon_{g}$.

\section{Numerical results}

In this section we show how to enable a nanophotonic structure computational design, generating reflections as small as possible. We formulate our problem as IP and reconstruct a function $\varepsilon(x)$ inside a domain $D_{F E M}$ using the adaptive optimization algorithm of [4]. Our computational set-up is the same as in [4]. As initial guess $\varepsilon_{g}(x)$ we take different constant values of the function $\varepsilon(x)$ inside the domain to be designed, on the coarse non-refined mesh, and we take $\varepsilon(x)=1.0$ everywhere else in $D$. We choose three different constant values of $\varepsilon_{g}(x)=\{0.5,1.5,2.0\}$ in (5). Our tests show that designed structures are sensitive to the choice of the initial guess $\varepsilon_{g}(x)$, and all guesses on the interval $\varepsilon_{g} \in[0.5,1.5]$ gives significant reduction of reflections. Figure 1 presents zoomed views of final designed domains obtained on a three times locally adaptively refined mesh.

\section{REFERENCES}

[1] L. Beilina, Adaptive hybrid FEM/FDM methods for inverse scattering problems. Inverse Problems and Information Technologies, V.1, N.3, pp.73-116, 2002.

[2] L. Beilina, Domain decomposition finite element/finite difference method for the conductivity reconstruction in a hyperbolic equation, Communications in Nonlinear Science and Numerical Simulation, Elsevier, 37, pp.222-237, 2016.

[3] L. Beilina, M. Cristofol and K. Niinimäki, Optimization approach for the simultaneous reconstruction of the dielectric permittivity and magnetic permeability functions from limited observations, Inverse Problems and Imaging, 9 (1), pp. 1-25, 2015.

[4] L. Beilina, L. Mpinganzima and P. Tassin, Computational design of nanophotonic structure using an adaptive finite element method, arXiv:1606.01737, 2016.

[5] S. C. Brenner and L. R. Scott, The Mathematical Theory of Finite Element Methods, Springer-Verlag, Berlin, 1994.

[6] Joannopoulos, Johnson, Winn and Meade, Photonic Crystals: Molding the Flow of Light, Second edition, Princeton Univ. Press, 2008.

[7] O. A. Ladyzhenskaya, Boundary Value Problems of Mathematical Physics, Springer-Verlag, Berlin, 1985.

[8] Maier, Plasmonics: Fundamentals and Applications, Springer, 2007.

[9] N. T. Thành, L. Beilina, M. V. Klibanov and M. A. Fiddy, Reconstruction of the refractive index from experimental backscattering data using a globally convergent inverse method, SIAM J. Scientific Computing, 36 (3), pp.273-293, 2014.

[10] Soukoulis, Wegener, Nature Photon. 5, 523, 2011. 